\title{
Short Gommunications
}

\section{Leaf Extract Purification by Silver Nitrate/Silica Gel Thin-Layer Chromatography}

\author{
By J. A. Zabkiewicz, R. A. B. Keates and C. J. W. Brooks \\ Department of Chemistry, University of Glasgow
}

(Received 1 August 1968)

In the half decade since $\mathrm{AgNO}_{3} /$ silica gel chromatography was introduced, the technique has found wide application in the lipid and steroid fields of research. The complex-forming abilities of $\mathrm{Ag}^{+}$ion have been used to enhance separations between closely related compounds, particularly those differing only in double-bond character. Though crystalline and stable $\mathrm{AgNO}_{3}$ complexes with organic compounds have been made, the method has not been used in chromatography to form complexes that are immobile in a variety of solvent systems. We now describe such a technique, which has been used by us in the preliminary separation of lipophilic material extracted from leaves.

The methods used for the separation of plant leaf extracts are rather varied. One of the complicating factors in any procedure is the inevitable presence of several chlorophylls and carotenoids in an extract. These compounds have various polarities, so that in a chromatographic separation of leaf extracts the isolation of a representative sterol or sesquiterpenoid fraction, clear of these contaminants, is rather difficult. In particular in studies of the incorporation of isotope-labelled biosynthetic intermediates it is desirable to isolate all the labelled sterols and lowor-molecular-weight terpenoids as a group, so that relative incorporations may be estimated by radioautography or other radioactivity-monitoring systems. This purified fraction should then be amenable to direct gasliquid-chromatographic examination without contamination by pyrolysis products of the carotenoids and chlorophylls. This last objective was a prime consideration, as the gas-liquidchromatographic examination of these extracts was essential to the analytical method employed.

It is well established that, with $\mathrm{AgNO}_{3}$-impregnated silica gel, retention depends on the degree of unsaturation in the molecule (Morris, 1966). This also holds for an improved version of the method (Wood \& Snyder, 1966). Accordingly the highly unsaturated carotenoids, even the non-oxygenated ones, and the chlorophylls should be effectively immobile in the usual organic solvents during either column or thin-layer chromatography. By this method, it should be possible to obtain an extract from which these compounds have been removed.

Thin-layer silica gel $\mathrm{G}$ plates impregnated with $10 \% \mathrm{AgNO}_{3}$ (by wt.) were prepared in the usual fashion (Morris, 1966). After drying overnight at $60^{\circ}$, the plates were stored in the dark over silica gel as desiccant and were not further activated before use.

Initial trials were conducted with a carotene extract from carrots (mainly $\beta$-carotene; Goodwin, 1955). When the plate was developed immediately after material had been applied at the origin, little difference was observed in $R_{F}$ values between a silica gel G plate and one impregnated with $\mathrm{AgNO}_{3}$. However, if the latter was left for an interval of $\frac{1}{2}-1 \mathrm{hr}$. (at about $40^{\circ}$ ) between application and elution, the carotenoid material formed a complex with the silver at the origin and did not move from it on subsequent elution with ethyl acetate-light petroleum (b.p. $\left.60-80^{\circ}\right)(3: 17, v / v)$. Whether the carotenoid material is irreversibly changed in this process has not been determined. On silica gel alone this delay makes no difference, apart from the usual slight increase in decomposition of the carotenoid material.

When a leaf extract (benzene solution, containing a variety of terpenoids) was run in a similar fashion, it was observed that the chlorophylls were also congregated at the origin, so that above $R_{F} 0 \cdot 1$ the only compounds present were terpenoids such as the sterols and sesquiterpenoids together with any other relatively saturated compounds present in the extract. Detection of these constituents after thin-layer chromatography was possible by using either ceric ammonium nitrate or an ethanolic solution of the sodium salt of 3-hydroxypyrene5,8,10-trisulphonic acid (Tschesche, Biernoth \& Wolff, 1963), a non-destructive fluorescent spray reagent that is considered by us superior to fluorescein or 2,7-dichlorofluorescein.

As further confirmation of the efficacy of the method, bands were scraped off the $\mathrm{AgNO}_{3} /$ silica 

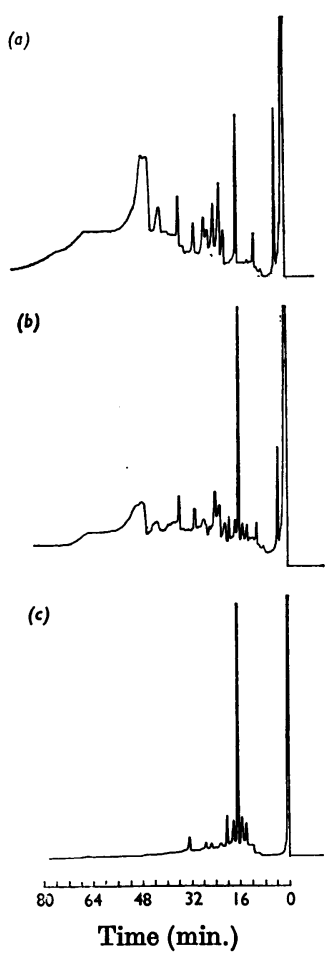

Fig. 1. Representative traces from the gas-liquid chromatography of: (a) carotenoid material from carrot; $(b)$ extract of 'carotenoid' band from a thin-layer chromatographic plate, containing both lower terpenoid and carotenoid material; (c) extract from a corresponding section of an $\mathrm{AgNO}_{3} /$ silica gel plate after complexes of carotenoid material had been formed; the absence of carotenoid decomposition fragments should be noted. Conditions for gas-liquid chromatography were: $3 \% \mathrm{SE}-30,5 \mathrm{ft} . \times \frac{1}{4}$ in. external-diam. glass dual columns, flow $60 \mathrm{ml} . / \mathrm{min}$., temperature programmed from $100^{\circ}$ to $250^{\circ}$ at $4^{\circ} / \mathrm{min}$., injector temperature $250^{\circ}$.

gels plates in the region in which carotenoids were known to occur if not present as complexes beforehand. This silica gel was extracted with dry benzene and the concentrated extract examined by gasliquid chromatography. Representative gas-liquidchromatographic traces are shown in Fig. 1. Fig. $1(a)$ shows the general appearance of a trace when carotenoid material alone is injected. A variety of peaks and a general base-line rise is observed. An extract of the carotenoid region from a thin-layer-chromatographic plate in which the $\mathrm{AgNO}_{3}$ has not been allowed to form complexes with the polyunsaturated components of a leaf extract is shown in Fig. $1(b)$. The terpenoid material is eluted first, but again a series of peaks and base-line rise is observed at longer retention times. Fig. $1(c)$ shows the absence of these later peaks and the improvement in base line (indicating that there is no slow elution of decomposition products) after a sample has been purified by the method described. It has been found that material in any part of the region on a thin-layer-chromatographic plate with $R_{F}$ greater than 0.1 can be assayed by gas-liquid chromatography directly after $\mathrm{AgNO}_{3} /$ silica gel thin-layer chromatography without interference by contaminating substances.

This technique has been used in the analysis of the radioactivity associated with sesquiterpenoids and sterols, and no inexplicable background radioactivity has been observed in any samples so far studied, nor have the minor peaks on gas-liquid chromatography of a sample been obscured by the pyrolysis fragments of carotenoid or chlorophyll material.

We thank Professor R. A. Raphael, F.R.S., for his encouragement. We are also indebted to Arthur Guinness Son and Co. (Dublin) Ltd. for a grant (to C.J.W.B.) that has aided this work.

Goodwin, T. W. (1955). In Modern Methods of Plant Analysis, Vol. 3, p. 279. Ed. by Paech, K. \& Tracey, M. V. Berlin: Springer-Verlag.

Morris, L. J. (1966). J. Lipid Res. 7, 717.

Tschesche, R., Biernoth, G. \& Wolff, G. (1963). J. Chromat. $12,342$.

Wood, R. \& Snyder, F. (1966). J. Amer. Oil Chem. Soc. 43, 53. 